\title{
The disk-bearing young star IM Lupi ${ }^{\star}$ X-ray properties and limits on accretion
}

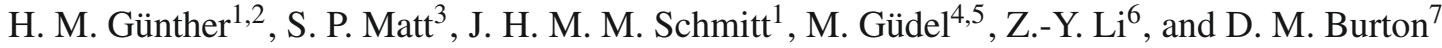 \\ 1 Hamburger Sternwarte, Universität Hamburg, Gojenbergsweg 112, 21029 Hamburg, Germany \\ e-mail: moritz.guenther@hs . uni-hamburg.de \\ 2 Harvard-Smithsonian Center for Astrophysics, 60 Garden Street, Cambridge, MA 02138, USA \\ 3 NASA Ames Research Center, Moffett Field, CA 94035, USA \\ 4 Institute of Astronomy, ETH Zürich, 8093 Zürich, Switzerland \\ 5 Department of Astronomy, University of Vienna, Türkenschanzstr. 17, 1180 Vienna, Austria \\ 6 Department of Astronomy, University of Virginia, PO Box 400325, Charlottesville, VA 22904, USA \\ 7 University of Southern Queensland, Toowoomba Qld 4350, Australia
}

Received 9 March 2010 / Accepted 13 May 2010

\section{ABSTRACT}

\begin{abstract}
Context. Classical T Tauri stars (CTTS) differ in their X-ray signatures from older pre-main sequence stars, e.g., weak-lined TTS (WTTS). CTTS exhibit a soft excess and deviations from the low-density coronal limit in the He-like triplets.

Aims. We test whether these features correlate with either accretion or the presence of a disk by observing IM Lup, a disk-bearing object apparently in transition between CTTS and WTTS without obvious accretion.

Methods. We analyse a Chandra grating spectrum and additional XMM-Newton data of IM Lup and accompanying optical spectra, some of which where taken simultaneously with the X-ray observations. We fit the X-ray emission lines and decompose the H $\alpha$ emission line into different components.

Results. In X-rays, IM Lup has a bright and hot active corona, where elements with low first-ionisation potential are depleted. The He-like Ne IX triplet is in the low-density state, but because of the small number of counts in the data a high-density scenario cannot be excluded at the $90 \%$ confidence level. In terms of all its X-ray properties, IM Lup resembles a main-sequence star, but is also compatible with CTTS signatures at the $90 \%$ confidence level, thus we cannot decide whether the soft excess and deviations from the low-density coronal limit for the He-like triplets in CTTS are produced by accretion or only the presence of a disk. The star IM Lup is chromospherically active, which accounts for most of its emission in $\mathrm{H} \alpha$. Despite its low equivalent width, the complexity of the $\mathrm{H} \alpha$ line profile is reminiscent of CTTS. We estimate the mass accretion rate to be $10^{-11} M_{\odot} \mathrm{yr}^{-1}$.
\end{abstract}

Key words. stars: formation - stars: individual: IM Lup - X-rays: stars

\section{Introduction}

Our understanding of the formation of stars and planetary systems and their emergence from molecular clouds has improved significantly during the past few decades. Stars form when giant molecular clouds fragment and contract to create proto-stars. Mass accretion onto those stellar cores proceeds by means of an accretion disk, while the surrounding envelope eventually disperses. The low-mass pre-main sequence stars at this stage are of two types, the classical T Tauri stars (CTTS) and the weak-lined $\mathrm{T}$ Tauri stars (WTTS). Traditionally, they were distinguished only in terms of their $\mathrm{H} \alpha$ emission-line equivalent width, stars with an $E W>10 \AA$ being defined to be CTTS. The $E W$ of $\mathrm{H} \alpha$ was found to be a good tracer of the accretion flow from the circumstellar disk of the CTTS. The $\mathrm{H} \alpha E W \mathrm{~s}$ of WTTS are quite small and their line profiles are symmetric; in contrast, CTTS exhibit broader emission lines, which are sometimes asymmetric (Fang et al. 2009). The combination of strong $\mathrm{H} \alpha$ emission

^ Based on observations obtained with XMM-Newton, an ESA science mission, and Chandra, a NASA science mission, both with instruments and contributions directly funded by ESA Member States and NASA. and line asymmetry is a reliable accretion indicator (Muzerolle et al. 1998, 2003).

Because of their low $\mathrm{H} \alpha E W$, WTTS are generally expected not to show accretion. The WTTS may indeed be more evolved CTTS, in which accretion has already stopped. This does not necessarily indicate the absence of a disk, as transitional disks may exist without ongoing accretion for some time (Padgett et al. 2006). For many TTS, the $\mathrm{H} \alpha E W$ is known to be variable, possibly because the accretion switches on and off or - at least - the accretion rate changes.

Both types of TTS have been known for a long time to be copious X-ray emitters (Feigelson \& Montmerle 1999). As a class, CTTS can be differentiated from other X-ray sources in terms of their strong soft X-ray excess (Robrade \& Schmitt 2007; Güdel \& Telleschi 2007), but CTTS also exhibit normal coronal activity and stellar flares.

The CTTS TW Hya, observed with Chandra/HETGS and XMM-Newton (Kastner et al. 2002; Stelzer \& Schmitt 2004), was the first star for which unusual line ratios in the He-like triplets were found, which are indicative of high densities in the formation region. This phenomenon has been detected in most CTTS observed so far (BP Tau: Schmitt et al. 2005; 
V4046 Sge: Günther et al. 2006; RU Lup: Robrade \& Schmitt 2007; MP Mus: Argiroffi et al. 2007; Hen 3-600: Huenemoerder et al. 2007). There are exceptions to this rule - in the more massive, eponymous $\mathrm{T}$ Tau itself, although known for its high accretion rate, the $\mathrm{O}$ VII triplet is consistent with the line ratio in the coronal limit (Güdel et al. 2007b). The same has been found for HAeBe stars, which are in a similar evolutionary state as the CTTS, yet of higher mass (AB Aur: Telleschi et al. 2007c; HD 163296: Günther \& Schmitt 2009). The soft excess and the He-like triplet line ratios in CTTS can be naturally linked to mass accretion from proto-planetary disks (Lamzin 1998; Günther et al. 2007).

Systems containing typical WTTS such as TWA 4 (Kastner et al. 2004) and TWA 5 (Argiroffi et al. 2005) show evidence of neither active accretion nor high densities in their X-ray spectra. Well-studied examples of young main-sequence (MS) stars such as AU Mic, Speedy Mic and AB Dor (Ness et al. 2004) also show triplets indicating low densities similar to older MS objects. Five of 83 WTTS observed by Padgett et al. (2006) show an IR-excess caused by dust within a few AU of the star, detected by Spitzer, thus there is a matter reservoir for accretion.

Observationally, we have been unable to determine whether the X-ray signatures observed in CTTS compared to WTTS and young MS stars are caused by active accretion or the presence of a disk. To solve this question, we observed a system with Chandra that was previously classified as a disk-bearing, nonaccreting WTTS. Simultaneously with the X-ray observations, the accretion state was observed using optical spectroscopy.

The properties of our target IM Lup are summarised in Sect. 2. We then present the X-ray observations of IM Lup and the accompanying optical data in Sect. 3. We show our new results and compare IM Lup to CTTS, WTTS, and MS stars in Sect. 4. In Sect. 5, we discuss the implications and end with a short summary in Sect. 6.

\section{Properties of IM Lup}

We present new observations of IM Lup (=Sz 82), the only known X-ray bright transition object with a small $\mathrm{H} \alpha E W(4 \AA)$ (Wichmann et al. 1999) that is far smaller than typical values for CTTS such as BP Tau and TW Hya. The IR-excess in IM Lup is indicative of a disk (Padgett et al. 2006), which has made it a favourite object for disk evolution studies in the IR (Padgett et al. 2006; Schegerer et al. 2006).

There is strong evidence that IM Lup is a single object from both ground-based speckle observations (Ghez et al. 1997) and space-based HST data (Pinte et al. 2008). Wichmann et al. (1998) derived a HIPPARCOS distance of $190 \pm 27 \mathrm{pc}$, greater than previous estimates but consistent with them within the errors. We apply this value throughout the paper. The spectral type of the star is M0, although its mass remains unclear. Values depend on the pre-main sequence evolutionary tracks adopted: based on the data of Hughes et al. (1994), the models of D'Antona $\&$ Mazzitelli (1994) yield $M=0.29 M_{\odot}$ and the models of Siess et al. (2000) indicate that $M=0.52 M_{\odot}$. Pinte et al. (2008) use the tracks from Baraffe et al. (1998) to infer that $M=1 M_{\odot}$ and $R=3 R_{\odot}$. The age is around $1 \mathrm{Myr}$ according to all models. Observationally, there is support for a mass of $M=1.2 \pm 0.4 M_{\odot}$ from millimetre observations, which resolve the emission lines from a disk in Keplerian rotation; the inclination of this disk is $54^{\circ} \pm 3^{\circ}$ (Panić et al. 2009). The reddening has been derived to be between $A_{V}=0.5$ (Pinte et al. 2008) and $A_{V}=1$ (Hughes et al. 1994).
Table 1. Observing log of X-ray observations.

\begin{tabular}{lllr}
\hline \hline Observatory & ObsID & Obs. date & Exp. time \\
\hline XMM-Newton & 0303900301 & $2005-08-08$ & $30 \mathrm{ks}$ \\
XMM-Newton & 0303900401 & $2005-08-17$ & $27 \mathrm{ks}$ \\
XMM-Newton & 0303900501 & $2005-09-06$ & $27 \mathrm{ks}$ \\
Chandra & 9938 & $2009-05-04$ & $150 \mathrm{ks}$ \\
\hline
\end{tabular}

The inner disk of IM Lup is resolved down to $<0.4 \mathrm{AU}$, dust grains have partially settled in the disk-midplane and grown to a few millimetres; the disk surface shows strong silicate emission bands and there are signatures of a low degree of crystallisation (Pinte et al. 2008). Beyond $400 \mathrm{AU}$, the surface density of $\mathrm{CO}$ gas and dust grains with sizes $1 \mu \mathrm{m}-1 \mathrm{~mm}$ declines dramatically but remains detectable out to 900 AU (Panić et al. 2009).

\section{Observations and data reduction}

\subsection{X-ray observations}

IM Lup was observed off-axis three times with XMM-Newton for a total exposure time of $84 \mathrm{ks}$ and with Chandra for $150 \mathrm{ks}$ in a dedicated observation using the high-energy transmission grating (HETGS). The observation log is summarised in Table 1. All data were reduced using either the standard XMM-Newton Science Analysis System (SAS) software, version 9.0, (Gabriel et al. 2004) or the Chandra Interactive Analysis of Observations (CIAO) software, version 4.1.2 (Fruscione et al. 2006), in the case of the Chandra observation. The XMM-Newton observations were contaminated by proton flares and we applied the standard selection criteria. We extracted CCD spectra from the PN and the MOS2 detector data. During the observations, one of the chips in the MOS1 camera did not operate and, unfortunately, IM Lup is located in this gap for two of the three observations. We primarily used the MOS2 data to compile our lightcurves because it is less affected by the proton noise, and the PN data for spectra because of their higher signal. We extracted lightcurves and CCD spectra for all three observations. We did not reprocess the Chandra evt2 file, thus the predefined selection criteria apply. Positive and negative orders for the HEG and MEG were merged. Spectral fitting was carried out using XSPEC V12.5.0 (Arnaud 1996), and individual line fluxes were measured using the CORA line fitting tool (Ness \& Wichmann 2002). CORA employs a maximum likelihood method, taking the Poisson statistics into account. Because the line widths are dominated by instrumental broadening, we kept them fixed at $\Delta \lambda=0.02 \AA$ for the MEG and $\Delta \lambda=0.01 \AA$ for the HEG, and the line profile was represented by a modified Lorentz profile with $\beta=2.5$. We fixed the wavelengths to the theoretical values taken from the CHIANTI 5.1 database (Dere et al. 1997; Landi et al. 2006). By fitting the strongest lines with a free wavelength we verified that our wavelength scale is accurate. The error introduced by fixing the wavelength is negligible compared to the uncertainties from counting statistics in all cases.

\subsection{Optical observations}

Observations were carried out using the $79 \mathrm{~g} / \mathrm{mm}$ echelle spectrograph $(R \approx 24000)$ on the Nasmyth-B focus of the Australian National University's $2.3 \mathrm{~m}$ telescope (ANU) on the nights of May 5th and 6th, 2009. Each night, two 1800 s exposures were taken one after another. The observing log for all optical observations is shown in Table 2. A GG490 filter was used in the beam 
Table 2. Log of optical observations.

\begin{tabular}{llllr}
\hline \hline No. & Observatory & ID & Obs. time & Exp. time \\
\hline H1 & ESO/HARPS & 6201 & 2008-05-09 02:19 & $20 \mathrm{~min}$ \\
H2 & ESO/HARPS & 6381 & $2008-05-0908: 55$ & $20 \mathrm{~min}$ \\
H3 & ESO/HAPRS & 6711 & $2008-05-1002: 18$ & $20 \mathrm{~min}$ \\
H4 & ESO/HARPS & 6971 & $2008-05-1008: 10$ & $20 \mathrm{~min}$ \\
A1 & ANU & 35 & $2009-05-0512: 38$ & $30 \mathrm{~min}$ \\
A2 & ANU & 36 & $2009-05-0513: 09$ & $30 \mathrm{~min}$ \\
A3 & ANU & 36 & $2009-05-0611: 50$ & $30 \mathrm{~min}$ \\
A4 & ANU & 37 & 2009-05-06 12:20 & $30 \mathrm{~min}$ \\
\hline
\end{tabular}

to avoid the overlapping of the first two orders at the reddest wavelengths. A cross disperser grating of $316 / 750 \mathrm{~nm}$ was used to allow collection of data of orders $55-34$ or $4120-6630 \AA$. Bias frames, ThAr Arc observations for wavelength calibration, and quartz lamp exposures for flat-fielding were also included to help perform the data reduction. The code used to reduce the $2.3 \mathrm{~m}$ data is a slightly modified version of ESpRIT (Echelle Spectra Reduction: an Interactive Tool) (Donati et al. 1997).

For comparison purposes, we also downloaded observations from the ESO $3.6 \mathrm{~m}$ telescope at La Silla obtained with HARPS for IM Lup, from the ESO archive (program ID 081.C-0779(A)). Public HARPS data packages have been released by the External Data Products (EDP) group within the Data Products department (DPD) at ESO. These HARPS advanced data products (ADP) have been produced by the automatic HARPS pipeline developed by the Observatoire Astronomique de l'Université de Genève $^{1}$. The uncertainty in the flux in these observations was assumed to be the Poisson error in the count statistic. This is consistent with an estimate from the spectra, where we chose an apparently line-free region close to the $\mathrm{H} \alpha$ line. We calculate the mean flux and the standard deviation. The relative error in the spectra is $3 \%$ for each bin in the oversampled spectrum, that is a signal-to-noise reatio $(S / N)$ of about 100 rebinned to instrumental resolution, except for the second exposure, where the relative error is $5 \%$.

To compare IM Lup to MS stars, we obtained spectra of HD 156274 and HD 156026 from the library of Bagnulo et al. (2003).

\section{Results}

In this section we show our observational results. We start with the lightcurves in Sect. 4.1, then we show data from the CCD detectors and models for these data in Sect. 4.2, before we concentrate on the line fluxes from high-resolution grating spectroscopy in Sect. 4.3. We finish with the optical spectra in Sect. 4.4.

\subsection{The $X$-ray lightcurve}

In Fig. 1, the X-ray lightcurve of IM Lup is shown. The XMM-Newton observations are separated by several days and IM Lup shows a steady increase in luminosity during this time, holding the ratios of hard $(1.5-10.0 \mathrm{keV})$ to soft $(0.3-1.5 \mathrm{keV})$ emission nearly constant. Because of different detector efficiencies, the separate hardness ratios of Chandra and XMM-Newton cannot be compared directly. During the much longer Chandra observation, there was small-scale variability in both bands, but without clear flaring activity. A KS-test shows that a constant

${ }^{1}$ http://archive.eso.org/archive/adp/ADP/HARPS/
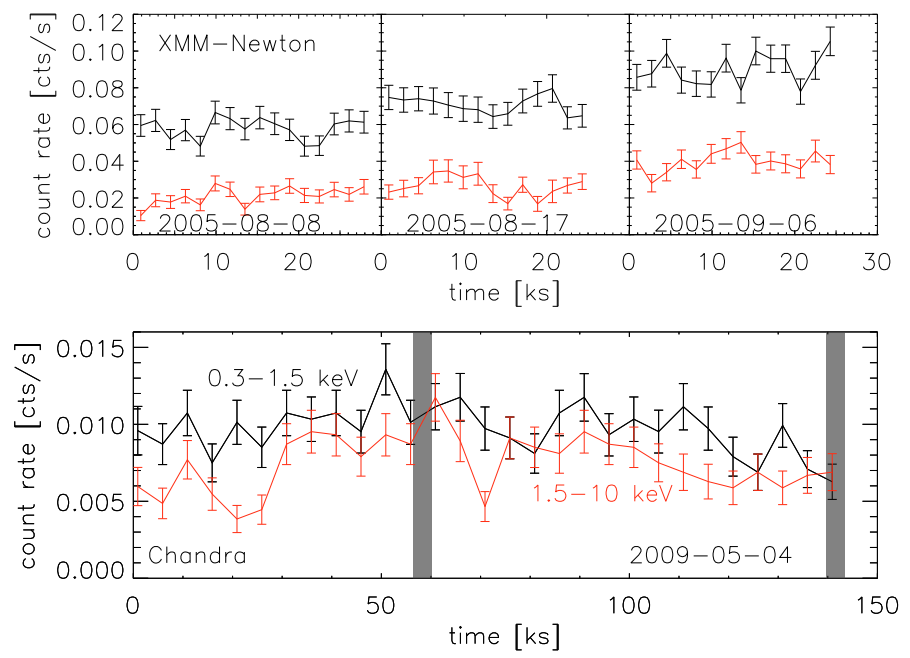

Fig. 1. Lightcurve in soft (black) and hard (red/grey) band. Because of the different detector efficiencies, the XMM-Newton and Chandra data cannot be compared directly. The shaded areas show the times of the optical observations.

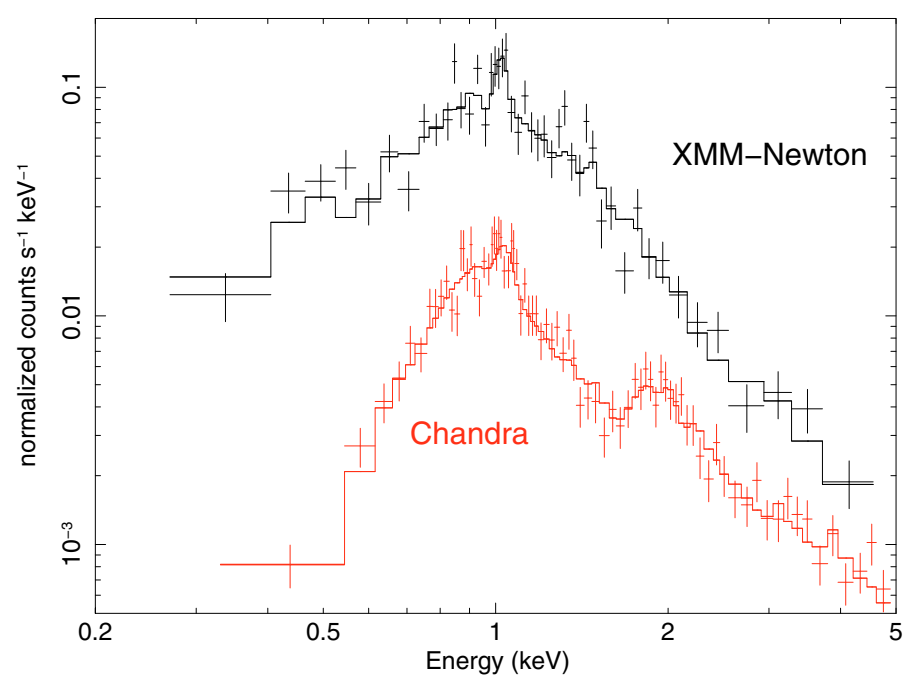

Fig. 2. CCD spectra of IM Lup from XMM-Newton EPIC/PN (ObsID 0303900401, upper curve) and Chandra ACIS (ObsID 9938, lower curve) with the best-fit model overlaid. See Table 3 for model parameters.

lightcurve can be rejected at the $72 \%$ level for the soft band and at the $98 \%$ level for the hard band.

\subsection{The CCD X-ray spectra}

In Fig. 2, we show the MOS2 CCD spectrum from the second XMM-Newton exposure and the zeroth order ACIS spectrum, both binned to a minimum of 25 counts per bin. At first sight, we can already identify the $\operatorname{NeX} \operatorname{Ly} \alpha$ line, which causes a peak around $1 \mathrm{keV}$ and the Si XIII and Si XIV emission around $2 \mathrm{keV}$. We describe the plasma in more detail with three optically thin emission components (vapec model) and a single photoabsorption model in XSPEC and overplot the best-fit model. A simpler model with only two components and tied absorbing column density $n_{\mathrm{H}}$, temperature, and abundances for all four observations already provides a statistically acceptable fit (red. $\chi^{2}=1.12$ ). However, the residuals show systematic trends at low energies, which is indicative of an inaccurate combination 
Table 3. Best-fit model for the CCD spectra ( $90 \%$ conf. ranges).

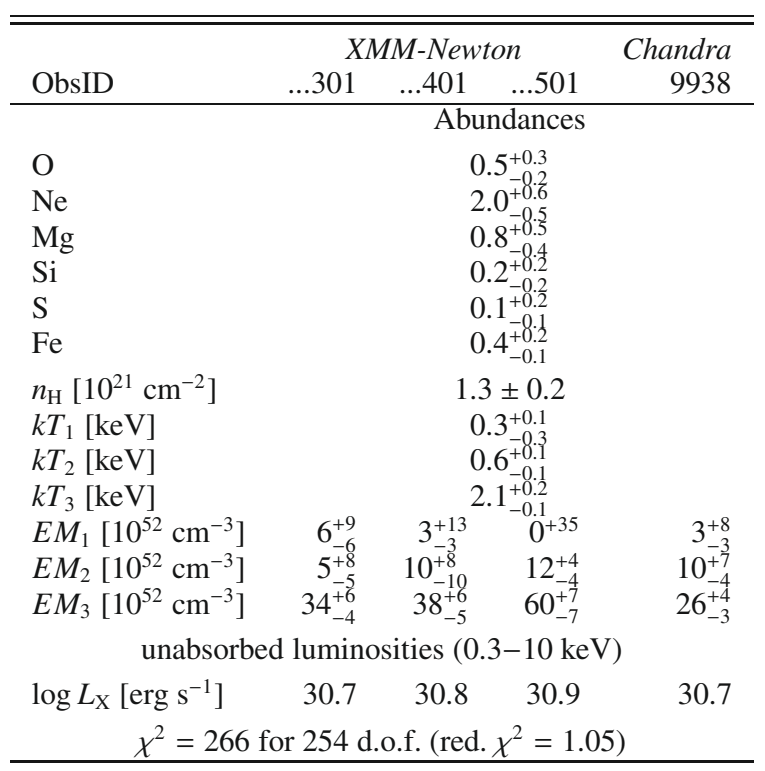

of $n_{\mathrm{H}}$ and $T_{1}$. We thus added a third vapec component, keeping the temperatures of the three components tied over all datasets. This ensured that the relative contributions of the soft, medium, and hard components can be compared. There is no significant difference in the fitted $n_{\mathrm{H}}$ between the separate datasets, thus we also keep it tied. The parameters of our best-fit model are given in Table 3 . The calculated errors represent $90 \%$ confidence ranges and the fitted abundances are relative to the solar values from Grevesse \& Sauval (1998); all other abundances are fixed at their respective solar values. Some of the values in the table are strongly correlated with each other. To maintain the overall luminosity of the star, when e.g., the soft component diminishes the emission measure $(E M)$ of the medium component has to be increased. In all observations, the emission is dominated by a hot plasma component around $k T=2.1 \mathrm{keV}$. The separation between the consecutive XMM-Newton exposures is 9 and 19 days. During this time, the total plasma emission measure increases by $60 \%$; at the same time, a larger portion of the total emission measure is concentrated at higher temperatures. The lightcurves in Fig. 1 show that this increase is steady during the observations. In the Chandra observation the distribution of the emission measure in the different components is comparable to the second XMM-Newton dataset, only the hot component being reduced by about a third; thus, the total emission measure is lower than in any of the XMM-Newton observations. We measured the absorbing column density towards the source as $N_{\mathrm{H}}=1.3 \pm 0.2 \times 10^{21} \mathrm{~cm}^{-3}$. We used this value to calculate the total unabsorbed $\mathrm{X}$-ray luminosities $L_{\mathrm{X}}$ given in Table 3. Assuming a standard gas-to-dust ratio, the optical reddening and the $\mathrm{X}$-ray absorption should be related with the formula $N_{\mathrm{H}}=A_{V} \cdot 2 \times 10^{21} \mathrm{~cm}^{-2}$ (Vuong et al. 2003), leading to $A_{V}=0.7 \mathrm{mag}$ in agreement with the values found by Hughes et al. (1994) and Pinte et al. (2008).

\subsection{The high-resolution X-ray spectra}

\subsubsection{Line fluxes}

The MEG grating spectrum is shown in Fig. 3. We measure line fluxes from both the HEG and MEG. In Table 4, we indicate the fluxes of all lines detected above the $2 \sigma$ level. In the case of the He-like triplets, we quote all lines, if one of them is strong enough to be included in the table. From the fitted line fluxes, we calculate the total line luminosity, using the absorption cross-section from Balucinska-Church \& McCammon (1992). The relative errors in the line intensity are larger than those in the line fluxes, because the uncertainty in the distance and the fitted $N_{\mathrm{H}}$ contribute to the total error budget.

We detect lines of $\mathrm{O}, \mathrm{Ne}, \mathrm{Mg}, \mathrm{Si}$, and Fe. The first four elements turn up in the Lyman series and in the He-like triplets; iron can be seen in the ionisation stages Fe XVII and Fe XVIII. Because of the low signal, only five lines can be detected in the HEG and all fluxes agree with the MEG measurement within the statistical uncertainty. In the following, we use only the MEG data.

A special analysis is required for the O VII He-like triplet, because its signal is very weak because of absorption and Chandra's low effective area in this region. Its flux is important for comparing IM Lup with other CTTS and MS stars. There are six photons recorded between $21.5 \AA$ and $22.2 \AA$ (at 21.60, $21.72,21.80,22.06,22.07$, and $22.10 \AA$ ), which is compatible with the background of 13 cts $\AA^{-1}$ determined from the linefree region from $20 \AA$ to $23 \AA$ around the O VII He-like triplet. However, for the line profile of Chandra, $88 \%$ of all photons are expected to be within one $F W H M=0.02 \AA$. Three of the six photons fall within this range of the theoretical wavelengths (21.6020, 21.8071, and 22.1012 $\AA$, according to the CHIANTI database), but only 0.25 photons are expected from the background in the central $F W H M$ of each line. According to Poisson statistics, 3 photons on a background of 0.75 photons represent a detection of O VII emission at the $95 \%$ significance level, but the uncertainty in the derived flux is obviously very large. The $90 \%$ upper limit to the flux, i.e., the flux for which there is a $10 \%$ probability of observing three or less photons in the O VII triplet is $2 \times 10^{-5} \mathrm{cts} \mathrm{s}^{-1} \mathrm{~cm}^{-2}$, which corresponds to a dereddened luminosity of $3 \times 10^{29} \mathrm{erg} \mathrm{s}^{-1}$.

\subsubsection{He-like triplets}

The He-like ions exhibit a triplet of lines, for which the line ratios are temperature and density sensitive. These triplets consist of a resonance $(r)$, an intercombination $(i)$, and a forbidden line ( $f$ ) (Gabriel \& Jordan 1969; Porquet et al. 2001). The $R$ - and $G$-ratios $(R=f / i$ and $G=(f+i) / r)$ are commonly used to describe the triplet; for high electron densities $n_{\mathrm{e}}$ or strong UV photon fields, the $R$-ratio falls below its low-density limit, because electrons are collisionally or radiatively excited from the upper level of the $f$ to the $i$ line, but the UV field of late-type stars such as IM Lup is far too weak to influence the $R$-ratio. We interpret the ratios in the context of the CHIANTI 5.1 database.

According to Table 4, the observed $R$-ratio for Si XIII is 1.8 , with a $90 \%$ lower limit of 1.0 according to a Monte Carlo simulation of Poisson-distributed counts using the observed count numbers as expectation values (for details see Günther \& Schmitt 2009, Appendix A). The predicted low-density limit for this ratio is 2.0 with smaller numbers for densities $\log n_{\mathrm{e}}<12.5$. Unsurprisingly, the Si XIII triplet is compatible with the low density limit (90\% range: $\left.\log n_{\mathrm{e}}<13.8\right)$. The G-ratio is temperature sensitive. For temperatures at which more than $10 \%$ of the $\mathrm{Si}$ is present in the form of Si XIII, its value is predicted to be between $1.2(\log T=6.1)$ and $0.6(\log T=7.2)$. This occurs in the $1 \sigma$ error range of the observations, thus the $G$-ratio does not constrain the models. 
H. M. Günther et al.: The disk-bearing young star IM Lupi

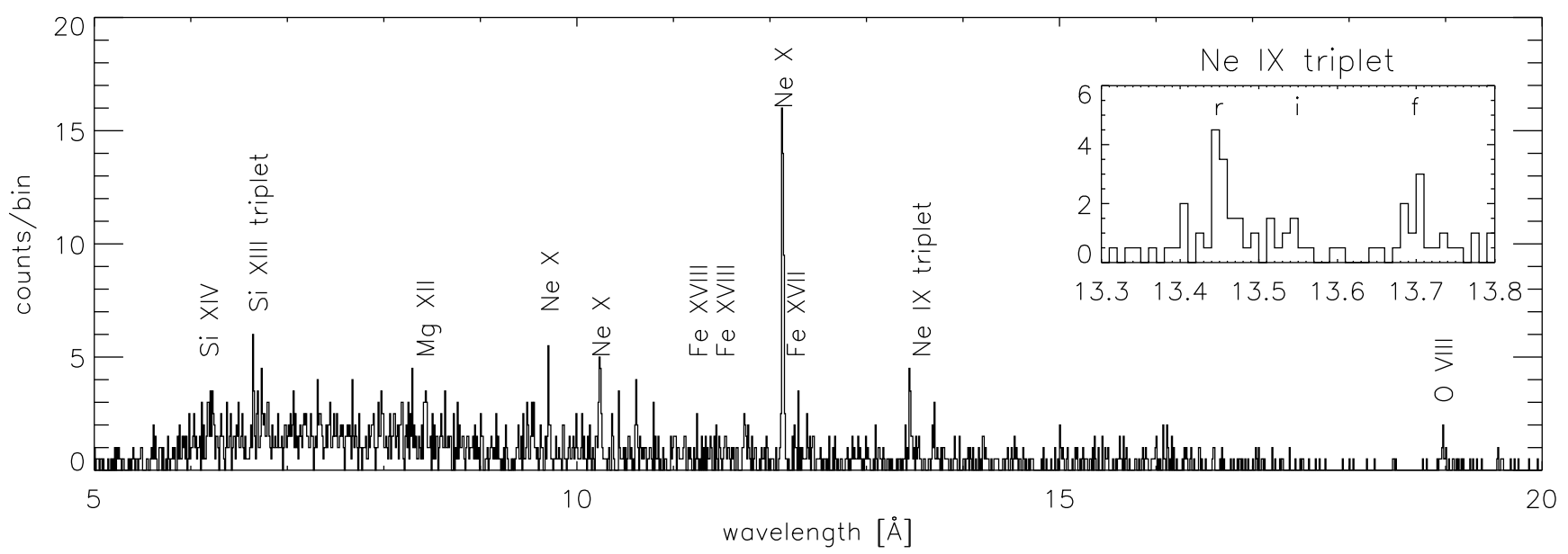

Fig. 3. MEG spectrum of IM Lup, rebinned by 2. The lines detected with more than $2 \sigma$ significance are marked (Table 4$)$. The inset shows an enlargement of the He-like Ne IX triplet.

Table 4. Measured line fluxes ( $1 \sigma$ errors).

\begin{tabular}{|c|c|c|c|c|}
\hline Ion & $\lambda[\AA]$ & Counts & Photon flux ${ }^{a}$ & Luminosity $^{b}$ \\
\hline \multicolumn{5}{|c|}{$-\mathrm{HEG}-$} \\
\hline Si XIV & 6.18 & $7.6 \pm 3.3$ & $1.2 \pm 0.5$ & $17 \pm 9$ \\
\hline Mg XII & 8.42 & $9.0 \pm 3.4$ & $1.5 \pm 0.6$ & $17 \pm 8$ \\
\hline $\mathrm{NeX}$ & 9.71 & $6.1 \pm 2.9$ & $1.8 \pm 0.8$ & $19 \pm 10$ \\
\hline $\operatorname{Ne} \mathrm{X}$ & 10.24 & $5.6 \pm 2.5$ & $2.6 \pm 1.2$ & $27 \pm 14$ \\
\hline $\mathrm{Ne} \mathrm{X}$ & 12.13 & $30.5 \pm 5.7$ & $20.3 \pm 3.8$ & $197 \pm 68$ \\
\hline \multicolumn{5}{|c|}{$-\mathrm{MEG}-$} \\
\hline Si XIV & 6.18 & $12.2 \pm 4.7$ & $0.9 \pm 0.3$ & $13 \pm 6$ \\
\hline Si XIII & 6.65 & $18.0 \pm 5.2$ & $1.2 \pm 0.4$ & $17 \pm 7$ \\
\hline Si XIII & 6.69 & $7.5 \pm 4.0$ & $0.5 \pm 0.3$ & $7 \pm 4$ \\
\hline Si XIII & 6.74 & $15.3 \pm 5.1$ & $0.9 \pm 0.3$ & $12 \pm 5$ \\
\hline Mg XII & 8.42 & $14.9 \pm 5.0$ & $0.9 \pm 0.3$ & $10 \pm 4$ \\
\hline $\mathrm{NeX}$ & 9.71 & $14.0 \pm 4.7$ & $1.4 \pm 0.5$ & $14 \pm 6$ \\
\hline $\mathrm{Ne} X$ & 10.24 & $22.6 \pm 5.7$ & $2.6 \pm 0.6$ & $26 \pm 10$ \\
\hline Fe XVIII & 11.25 & $6.7 \pm 3.0$ & $1.1 \pm 0.5$ & $11 \pm 6$ \\
\hline Fe XVIII & 11.53 & $8.9 \pm 3.4$ & $1.5 \pm 0.6$ & $15 \pm 7$ \\
\hline $\mathrm{Ne} \mathrm{x}$ & 12.13 & $90.8 \pm 9.7$ & $19.7 \pm 2.1$ & $191 \pm 59$ \\
\hline Fe XVII & 12.26 & $10.2 \pm 3.8$ & $2.3 \pm 0.9$ & $22 \pm 10$ \\
\hline Ne IX & 13.45 & $20.9 \pm 5.0$ & $7.1 \pm 1.7$ & $70 \pm 26$ \\
\hline $\mathrm{Ne} I \mathrm{X}$ & 13.56 & $3.3 \pm 2.6$ & $1.2 \pm 0.9$ & $12 \pm 10$ \\
\hline $\mathrm{Ne}$ IX & 13.70 & $10.9 \pm 3.9$ & $4.0 \pm 1.4$ & $40 \pm 18$ \\
\hline O VIII & 18.97 & $7.9 \pm 3.3$ & $9.9 \pm 4.1$ & $103 \pm 54$ \\
\hline
\end{tabular}

Notes. (a) In units of $10^{-6} \mathrm{~s}^{-1} \mathrm{~cm}^{-2}$; (b) unabsorbed, in units of $10^{27} \mathrm{erg} \mathrm{s}^{-1}$.

In the Ne IX triplet, the formal $f / i$-ratio is 3 , the Monte Carlo simulation providing lower boundaries to the ratio of $2(1 \sigma)$ and $0.8(90 \%)$. According to CHIANTI, the $f / i$ ratio in the low density regime is 2.8 , thus the respective limits to the electron density in the emitting region are $\log n_{\mathrm{e}}<11.4(1 \sigma)$ and $\log n_{\mathrm{e}}<12.3(90 \%)$. The intercombination line in the Ne IX triplet is known to be contaminated with $\mathrm{Fe}$ lines in many cases, which should increase the $f / i$ ratio, however, iron is underabundant in IM Lup. The Ne IX $r$ line also contains a blend from Fe XIX. We cannot correct for this blend because no single Fe XIX is detected and, given the low abundance of Fe, we estimate that this blend is far smaller than the observational uncertainty in the line flux.

In summary, the $R$-ratio of the Si XIII triplet limits the electron density of the emitting plasma to be $n_{\mathrm{e}}<13.8$, and the $R$-ratio of the Ne IX triplet limits the density to $\log n_{\mathrm{e}}<12.3$ ( $90 \%$ confidence in both cases).

\subsection{The optical spectra}

The optical spectra are dominated by photospheric absorption lines. From the high-resolution HARPS data, we measure the rotational broadening to be $v \sin i=15 \pm 2 \mathrm{~km} \mathrm{~s}^{-1}$ by comparison with synthetic PHOENIX spectra (Hauschildt \& Baron 1999). Using the stellar radius and the inclination determined by Pinte et al. (2008), this implies a stellar rotation period of 8 days, if the disk inclination and the stellar inclination agree. We compared the spectra to two templates taken from MS stars of similar spectral type (HD 156274 and HD 156026) in the library of Bagnulo et al. (2003). The spectra are broadened and degraded to the spectral resolution of our IM Lup data. The depth of most photospheric lines matches well, thus no significant veiling is present in IM Lup.

In the following, we discuss where IM Lup differs from the templates. We find Li I in absorption at $6708 \AA$, confirming its youth. The equivalent width of this line is about $-0.3 \AA$. Typical activity tracers such as $\mathrm{Ca} \mathrm{K}$ and the Balmer series are in emission. Figure 4 shows the $\mathrm{Ca} \mathrm{H}$ and $\mathrm{K}$ lines, and Fig. 5 the $\mathrm{H} \alpha$ line profile in the four different exposures taken simultaneously to the Chandra observation. The $\mathrm{H} \alpha$ equivalent width increases during our observations from $2.8 \AA$ on the first day (A1 and A2) to 4.5 (A3) and $5.2 \AA$ (A4) in the two separate exposures on the second day, respectively in terms of time, where we measured the emission core of the line only (in accordance with the X-ray data, we use the convention of denoting emission lines with a positive $E W$ and absorption lines with negative values).

In all cases, the $\mathrm{H} \alpha E W$ remains below $10 \AA$, which is the canonical dividing line between CTTS and WTTS. The line profile is asymmetric, with additional emission on the blue side. On the first day, a small bump exists at $+100 \mathrm{~km} \mathrm{~s}^{-1}$, which might be due to an unresolved secondary peak at this velocity. This seems to be the typical line profile for IM Lup; it was also observed by Wichmann et al. (1999). In a HARPS observation (Fig. 6), secondary peaks on both sides of the $\mathrm{H} \alpha$ line appear. On the second day of our ANU observations (A3 and A4), broad absorption wings are present, which are only marginally visible on the first day (A1 and A2), while at the same time the central emission is more strongly peaked. According to the classification scheme of Reipurth et al. (1996), the $\mathrm{H} \alpha$ line profile in 1998 is III-R (Wichmann et al. 1999), in 2008 III-B/R, and in 2009 III-R on the first day and IV-B/R on the second day. 


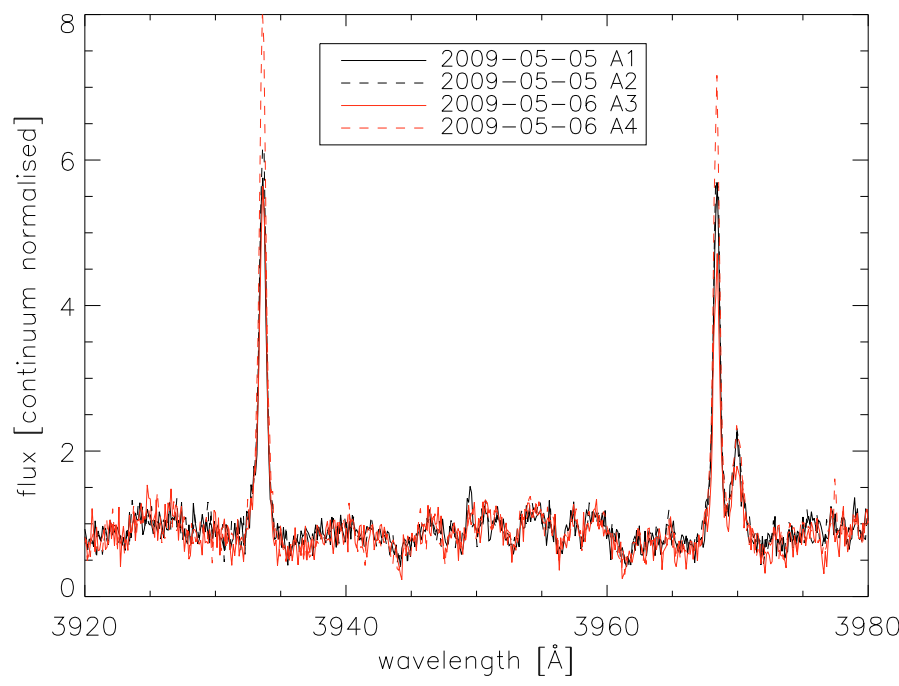

Fig. 4. $\mathrm{Ca} \mathrm{H}$ and $\mathrm{K}$ lines in all four exposures. For both lines, only sharp emission peaks are visible. The small line at $3970 \AA$ is $\mathrm{H} \epsilon$.

In addition to $\mathrm{H} \alpha$, the other detected Balmer lines and He I $5867 \AA$ increase in strength on the second day, in contrast to the X-ray emission, which weakens. Surprisingly, Fig. 1 shows that the X-ray count rate during the observations on the second day is only half of that on the first day; the hardness ratio remains constant.

For comparison, we analyse a HARPS dataset of four exposures of IM Lup, which was taken one year before the Chandra observations. The $\mathrm{H} \alpha$ emission line, shown in Fig. 6, looks similar; its total equivalent width (including the absorption component) decreases during the observations from $4.3 \AA$ to $2.7 \AA$. The spectral resolution in these observations is higher, thus we can reliably fit the separate components of the line. We exclude two narrow absorption lines from the profile fitting (Ti I and Fe I) and also ignore the centre of the $\mathrm{H} \alpha$ line, which is probably affected by the optical depth. In total, we find that five Gaussian components are necessary to describe the line profile. The most prominent component is the central emission peak, in addition to two narrow emission components shifted by about $100 \mathrm{~km} \mathrm{~s}^{-1}$ to the blue and red side, respectively, and two broad components, one of which is in absorption. The components are listed in Table 5 and shown in Fig. 6. Absorption below the continuum is seen only for velocities above $140 \mathrm{~km} \mathrm{~s}^{-1}$. The wings on the red side are most closely fit by a wide absorption component with a centroid close to the stellar rest velocity, when we attempt to fit a Gaussian shape. If the absorption on the red side is non-Gaussian, then the wide emission component is no longer needed and there is no absorption on the blue side of the line, so that the three narrow emission components are sufficient to describe the line shape. The central emission corresponds to the stellar rest velocity at all times, its amplitude changes with time. In the fit, this is evident as decreasing flux in the wide component, while the central component remains nearly constant. The narrow red-shifted component moves towards the centre during the observations, while the narrow, blue-shifted emission keeps its position.

In the ANU observations, a qualitatively similar structure is observed, but the resolution and signal-to-noise ratio is lower, so only two Gaussians are required. Absorption seen on both sides of the line on the second day of the ANU observations (A3 and A4 in Fig. 5) is consistent with a wide absorption component centred on the stellar rest velocity. This component is

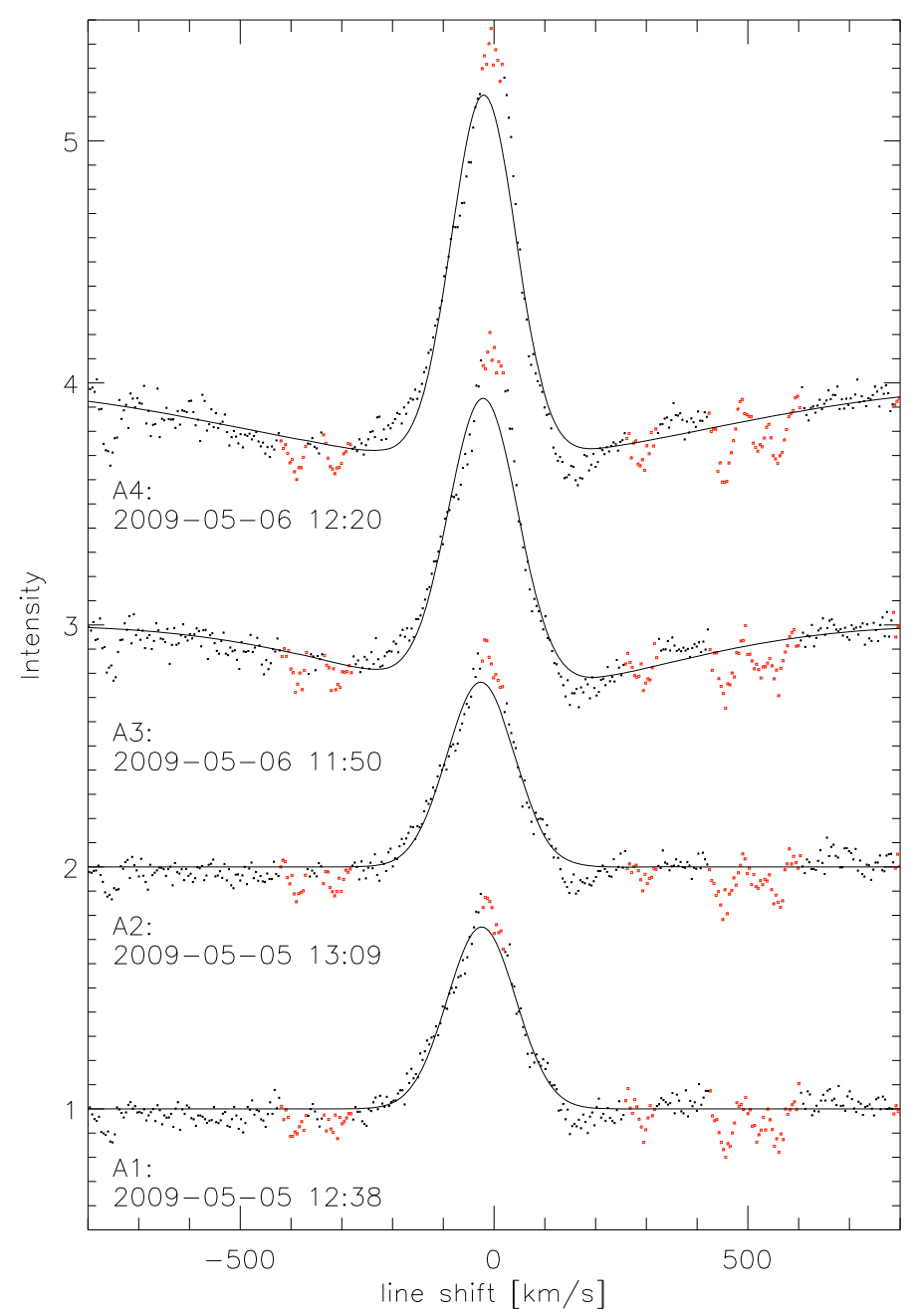

Fig. 5. $\mathrm{H} \alpha$ line profile in all four exposures. The line is in emission and asymmetric. A broad absorption component is also visible for the second day. Observations are continuum-normalised and shifted in intensity for clarity. The red/grey data points are excluded from the fit. Solid lines show a fit.

significantly broader than in the HARPS dataset. The FWHM of the main emission feature of $160 \mathrm{~km} \mathrm{~s}^{-1}$ and the velocity shift of the bump on the red side of the line profile in the first two observations agrees with the narrow red component in the HARPS observations (Table 5). The absorption in the ANU observations is stronger and broader than in the HARPS data, it being stronger than the emission line on the second day. Gaussian profiles are not ideal for fitting the line in Fig. 5, the fit is both too shallow and too broad on the red side around $150 \mathrm{~km} \mathrm{~s}^{-1}$, but they illustrate the change in relative strength between the observations. The fits underpredict the flux in the line center, because the line is more strongly peaked than a Gaussian.

\subsection{Comparison X-ray properties to CTTS, WTTS, and MS stars}

To compare the X-ray luminosity with other TTS of similar spectral type, we used the data published from the COUP (Preibisch et al. 2005) and the XEST (Güdel et al. 2007a) projects. Both are large X-ray surveys covering the star forming regions of Orion and the Taurus molecular cloud, respectively. Relative to the sources in both datasets IM Lup is a bright object. 
Table 5. Properties of line profile components.

\begin{tabular}{|c|c|c|c|c|}
\hline Comp. & No & $\begin{array}{r}E W^{a} \\
{[\AA]}\end{array}$ & $\begin{array}{r}\text { centre } \\
{\left[\mathrm{km} \mathrm{s}^{-1}\right]}\end{array}$ & $\begin{array}{r}F W H M \\
{\left[\mathrm{~km} \mathrm{~s}^{-1}\right]}\end{array}$ \\
\hline \multicolumn{5}{|c|}{ HARPS - fit with 5 Gaussians } \\
\hline \multirow[t]{4}{*}{ central } & H1 & $3.9 \pm 0.1$ & $-2.6 \pm 0.2$ & $76 \pm 1$ \\
\hline & $\mathrm{H} 2$ & $3.6 \pm 0.2$ & $-1.7 \pm 0.4$ & $77 \pm 2$ \\
\hline & $\mathrm{H} 3$ & $3.9 \pm 0.1$ & $-5.7 \pm 0.2$ & $85 \pm 1$ \\
\hline & $\mathrm{H} 4$ & $3.9 \pm 0.1$ & $-4.2 \pm 0.3$ & $82 \pm 1$ \\
\hline \multirow[t]{4}{*}{ blue wide } & H1 & $3.4 \pm 1.1$ & $-62 \pm 9$ & $248 \pm 13$ \\
\hline & $\mathrm{H} 2$ & $4.4 \pm 3.8$ & $-53 \pm 22$ & $249 \pm 30$ \\
\hline & $\mathrm{H} 3$ & $0.8 \pm 0.1$ & $-133 \pm 3$ & $115 \pm 4$ \\
\hline & $\mathrm{H} 4$ & $1.5 \pm 0.4$ & $-128 \pm 7$ & $166 \pm 10$ \\
\hline \multirow[t]{4}{*}{ blue narrow } & H1 & $0.34 \pm 0.02$ & $-92.2 \pm 0.6$ & $51 \pm 2$ \\
\hline & $\mathrm{H} 2$ & $0.29 \pm 0.04$ & $-90.5 \pm 1.2$ & $43 \pm 4$ \\
\hline & $\mathrm{H} 3$ & $0.55 \pm 0.02$ & $-95.6 \pm 0.4$ & $49 \pm 1$ \\
\hline & $\mathrm{H} 4$ & $0.62 \pm 0.04$ & $-95.5 \pm 0.5$ & $54 \pm 2$ \\
\hline \multirow[t]{4}{*}{ wide abs } & H1 & $-3.9 \pm 2.3$ & $-12 \pm 4$ & $412 \pm 24$ \\
\hline & $\mathrm{H} 2$ & $-4.6 \pm 5.3$ & $-5 \pm 16$ & $354 \pm 43$ \\
\hline & $\mathrm{H} 3$ & $-2.2 \pm 0.3$ & $36 \pm 3$ & $312 \pm 6$ \\
\hline & $\mathrm{H} 4$ & $-3.8 \pm 0.7$ & $7 \pm 4$ & $351 \pm 7$ \\
\hline \multirow[t]{5}{*}{ red narrow } & $\mathrm{H} 1$ & $0.61 \pm 0.04$ & $100.6 \pm 0.5$ & $60 \pm 2$ \\
\hline & $\mathrm{H} 2$ & $0.51 \pm 0.09$ & $96.3 \pm 1.4$ & $60 \pm 5$ \\
\hline & $\mathrm{H} 3$ & $0.48 \pm 0.01$ & $93.8 \pm 0.4$ & $55 \pm 1$ \\
\hline & $\mathrm{H} 4$ & $0.47 \pm 0.03$ & $86.2 \pm 0.9$ & $62 \pm 2$ \\
\hline & AN & - fit with 1 & 2 Gaussians & \\
\hline \multirow[t]{4}{*}{ central } & A1 & $2.8 \pm 0.2$ & $-25.8 \pm 1.0$ & $159 . \pm 3$. \\
\hline & A2 & $2.8 \pm 0.2$ & $-27.5 \pm 1.0$ & 157. \pm 2 \\
\hline & A3 & $4.5 \pm 0.2$ & $-22.6 \pm 0.6$ & 161. \pm 2 \\
\hline & A4 & $5.2 \pm 0.2$ & $-21.7 \pm 0.5$ & $150 . \pm 1$ \\
\hline \multirow[t]{2}{*}{ absorption } & A3 & $-4.7 \pm 2.8$ & $15 . \pm 7$ & $750 . \pm 50$ \\
\hline & A4 & $-7.8 \pm 5.1$ & $-36 . \pm 6$ & 1050. \pm 50 . \\
\hline
\end{tabular}

Notes. ${ }^{(a)}$ Absorption is shown as negative values.

Compared to the Taurus molecular cloud (Telleschi et al. 2007a), the mean temperature and X-ray luminosity $L_{X}$ of IM Lup implies that it is the most luminous CTTS. If it is a WTTS, it still belongs to the brightest $20 \%$ of all WTTS in that region.

The bolometric luminosity of IM Lup is $L_{\mathrm{bol}}=1.9 L_{\odot}$ (Pinte et al. 2008) so $\log \left(L_{\mathrm{X}} / L_{\mathrm{bol}}\right)=-3.1$. This number is typical of both MS stars, which saturate at $\log \left(L_{\mathrm{X}} / L_{\mathrm{bol}}\right)=-3$, and either CTTS or WTTS (Flaccomio et al. 2003; Preibisch et al. 2005).

The abundances of IM Lup shown in Table 3 exhibit an IFIP (inverse first ionisation potential) pattern, where elements of high first ionisation potential are enriched relative to the solar abundance and those of low FIP are depleted. This pattern is observed for most active stars, independent of their evolutionary state. The values measured for IM Lup agree with the typical abundances given by Güdel (2004).

Robrade \& Schmitt (2007) and Güdel \& Telleschi (2007) analyse the OVIII/O VII ratio as a measure of the excess of soft emission in CTTS with respect to stars on the MS. These studies illustrate that the observed excess is confined to a narrow temperature range on the formation of the He-like O VII triplet at 1-2 MK. The upper limit that we determine to the $\mathrm{O}$ VII emission in IM Lup is compatible with both MS stars and the soft excess in CTTS.

The $f / i$ ratio of the Ne IX triplet in IM Lup is statistically compatible at the $1 \sigma$ level with the typical values of coronal sources (Ness et al. 2004) at the low-density limit; however, a lower $f / i$ value is allowed for IM Lup at the $90 \%$ confidence level due to the low count statistic. The low-density limit is also observed in WTTS (TWA 4 Kastner et al. 2004; TWA 5

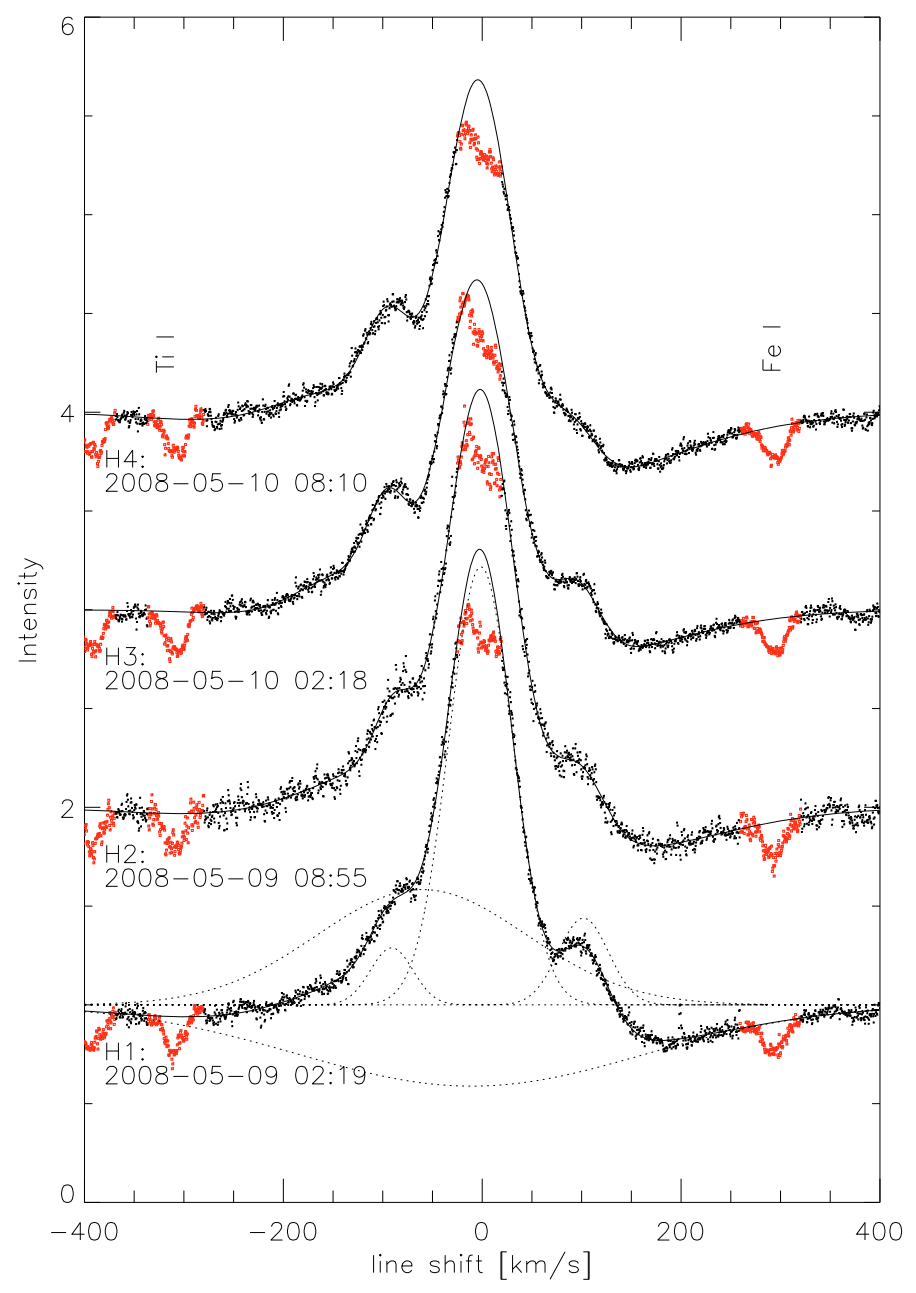

Fig. 6. $\mathrm{H} \alpha$ line profile in four HARPS exposures one year before our observations. Observations are continuum normalised and shifted in intensity for clarity. The red/grey data points are excluded from the fit. The lines show the best fit of five Gaussians given in Table 5. The single components are indicated for the first observation. Note the smaller velocity scale compared to Fig. 5.

Argiroffi et al. 2005; and HDE 245059 Baldovin-Saavedra et al. 2009). On the other hand, most CTTS show a relatively low $f / i$ ratio, which is attributed to accretion spots on their surface. TW Hya (Kastner et al. 2002; Stelzer \& Schmitt 2004), BP Tau (Schmitt et al. 2005), V4046 Sge (Günther et al. 2006), RU Lup (Robrade \& Schmitt 2007), MP Mus (Argiroffi et al. 2007), and Hen 3-600 (Huenemoerder et al. 2007) all show significant deviations from the low-density limit.

Going beyond the He-like triplets, we compared our MEG spectrum (Fig. 3) of IM Lup with a Chandra/MEG spectrum of YY Gem (Liefke et al. 2008), which is a MS binary of spectral type M3-M4, similar to IM Lup. After assuming the absorbing column density found for IM Lup, both spectra show the same features. The remaining small differences in the line fluxes are due to small abundance effects and IM Lup is a little brighter in the short-wavelength continuum, because of its strong, hot corona. Notable differences are found, when the same experiment is performed with the spectrum of TW Hya, because it lacks the strong emission component at $2.1 \mathrm{keV}$. 


\section{Discussion}

\subsection{X-ray properties}

IM Lup shows many characteristics of an active star. It has a bright, hot corona and its abundances follow the IFIP pattern, traits that are common to both CTTS and WTTS. In the density diagnostic of the He-like triplets, there is no deviation from the coronal limit, although because of the low signal a highdensity state is allowed at the $90 \%$ confidence level. The signal of the O VII triplet is too weak to check for any soft X-ray excess. Thus, we conclude that - from the X-ray point-of-view - it is more likely that IM Lup shares its characteristics with WTTS, despite the presence of its disk. If confirmed, this suggests that the distinctive characteristics of CTTS are really due to accretion, where an excess of soft X-ray emission originates in the high-density environment of an accretion spot as simulated by Lamzin (1998) and Günther et al. (2007). Our observation is incompatible with any hypothetical scenario where the high-density signatures of the He-like triplets are caused by the accretion disk itself because these signatures are not observed (at the $1 \sigma$ confidence level) in IM Lup, which has a disk.

In comparison to the WTTS samples of COUP and XEST, the only peculiarity is the high bolometric luminosity of IM Lup. It belongs to the brightest objects of its kind. This may be due to an overestimation of the distance. We use $190 \pm 27 \mathrm{pc}$ from Wichmann et al. (1998) based on the HIPPARCOS parallax, but other methods lead to lower values of $140 \pm 20 \mathrm{pc}$ (Hughes et al. $1993)$. Both values are compatible within the $2 \sigma$ errors, but if the lower value was the true one, the $\mathrm{X}$-ray emission measure and bolometric luminosity would be only half of the values given above. This does not change any of the other conclusions of our analysis, but IM Lup would represent the bulk of the COUP and XEST objects in this case. Nevertheless, IM Lup is an active star with a bright and hot corona and $\log \left(L_{\mathrm{X}} / L_{\text {bol }}\right)=-3.1$, similar to saturated MS stars and also typical of CTTS (Güdel \& Nazé 2009).

\subsection{Mass accretion?}

IM Lup has a high level of chromospheric activity, which causes the emission observed in $\mathrm{H} \alpha$. Other $\mathrm{M}$ dwarfs with active chromospheres also have $\mathrm{H} \alpha$ emission, typically with an equivalent width of a few $\AA$ (Worden et al. 1981; Robinson et al. 1990), that is similar to IM Lup. Their $\mathrm{Ca} \mathrm{H}$ and $\mathrm{K}$ lines often have very narrow emission peaks. The $\mathrm{H} \alpha$ line profiles vary, but in most active stars there is a single, narrow emission peak, sometimes with a reversal due to self-absorption at the centre. This is in line with the central peak in the IM Lup data. In the samples of Worden et al. (1981) and Robinson et al. (1990), there is not a single case in which $\mathrm{H} \alpha$ is in emission, where simultaneously broad absorption is present. The same is true for WTTS, which have narrow emission lines of symmetric shape (Fang et al. 2009).

In contrast, red-shifted absorption dips and red- and blueshifted emission components appear in accretion models for most viewing geometries (Muzerolle et al. 2001). Thus, in IM Lup the $\mathrm{H} \alpha$ line profile must be caused by material that is not present in MS stars, for instance a circumstellar envelope, the disk, or accretion funnels. We do not detect any veiling of the photospheric emission lines, thus any mass accretion must be very low, if present at all. The narrow emission components might originate in a weak accretion funnel. They are shifted by $\pm 100 \mathrm{~km} \mathrm{~s}^{-1}$, because we see accretion towards both the observer's side and the back side of the star. The line shift is smaller than usually seen in emission lines from accretion funnels of CTTS, but given the uncertain stellar parameters of IM Lup this could be due to a combination of low mass, i.e., lower gravitational potential, and viewing geometry, where the accretion funnel is not parallel to the line-of-sight. In some stars, the $\mathrm{H} \alpha$ line profile is also influenced by strong flares but this cannot be the case in IM Lup, because no flare shows up in the X-ray lightcurve.

White \& Basri (2003) attempted to find a correlation between the width of the $\mathrm{H} \alpha$ line at $10 \%$ of the maximum and accretion. They proposed a full width at $10 \%$ of the maximum $>270 \mathrm{~km} \mathrm{~s}^{-1}$ as a borderline between accreting and nonaccreting sources, a slightly lower limit of $>200 \mathrm{~km} \mathrm{~s}^{-1}$ being presented by Jayawardhana et al. (2003). The line width of IM Lup falls just between those two limits; it would be classified as non-accreting by one limit and as accreting by the other. Natta et al. (2004) showed that a quantitative relation between line width and mass accretion rate exists, which is valid from CTTS to sub-stellar objects. This relation yields an accretion rate of $10^{-11} M_{\odot} \mathrm{yr}^{-1}$, but IM Lup is chromospherically very active, which explains a large fraction of the $\mathrm{H} \alpha$ flux. Not all relations employed to convert the $\mathrm{H} \alpha$ flux to a mass accretion rate agree, if accretion is present at all, therefore we treat this number as a only rough estimate.

In summary, the $\mathrm{H} \alpha E W$ classifies IM Lup as a WTTS, but the $\mathrm{H} \alpha$ line profile is as complex as in CTTS. This suggests that mass accretion occurs at a very low rate. The energy released from accretion is too low to influence the X-ray properties significantly. For a mass accretion rate of $10^{-11} M_{\odot} \mathrm{yr}^{-1}$, at most $0.5 \%$ of the X-rays may be produced by accretion, assuming that $10 \%$ of the accretion energy is released in X-rays (Günther et al. 2007). For the interpretation of the X-ray data, we can thus safely treat IM Lup as a non-accreting source.

\subsection{Varying absorption?}

The HARPS H $\alpha$ line profiles are presented in Fig. 6. While the fitting with Gaussians (Table 5) yields an absorption component reaching over the entire line profile, for non-Gaussian components absorption is only required on the red side where the observed line profile falls below the continuum. The situation is different for the ANU data, which was obtained simultaneously to the Chandra observation. No absorption is visible here on the first day, and wide absorption wings are present on both sides of the line on the second day. We postulate that matter from the disk on its way onto the star causes the H $\alpha$ absorption. With IM Lup's mass and radius (Sect. 2), we calculate the Keplerian rotation of the disk. At the co-rotation radius of $6 R_{*}$, it matches the stellar rotation period calculated in Sect. 4.4; in this region, the inner disk should be truncated. The absorption component is strong at $100-400 \mathrm{~km} \mathrm{~s}^{-1}$, which corresponds to the free-fall velocity from the co-rotation radius. Thus the $\mathrm{H} \alpha$ absorbing gas may be found in the gap between star and disk; this scenario can only explain the red-shifted part of the absorption, but strengthens the argument in Sect. 5.2 that the $\mathrm{H} \alpha$ line width at $10 \%$ maximum may be caused by accretion. We speculate that the blue-shifted absorption in the ANU data is caused by an outflow. CTTS commonly drive winds or even jets, where the mass loss is of the order of $10 \%$ of the accretion rate (Cabrit et al. 1990; Coffey et al. 2008). The blue-shifted absorption appears only on the second day of the ANU observation, when the absorption on the red side, which we interpret as accretion, is stronger.

We divided the Chandra observation into two parts and fitted the $n_{\mathrm{H}}$ independently to test whether the absorption in the X-ray 
data changes with time. We found no change in the fitted value. The hardness ratio of the X-ray spectra does also not vary during the observations. This does not rule out variability in $n_{\mathrm{H}}$, but sets its upper limit. We estimate that an additional absorbing column of $>10^{21} \mathrm{~cm}^{-2}$ should have been detected. Does the accretion scenario explain why strong $\mathrm{H} \alpha$ absorption occurs with weak X-ray emission? Given the weak limit to the variability in $n_{\mathrm{H}}$ and that we have only two datapoints of simultaneous X-ray and optical spectra, the relation between $\mathrm{H} \alpha$ absorption and X-ray luminosity is - at best - only indicative. The lower X-ray luminosity and the $\mathrm{H} \alpha$ line profiles may, however, be caused by absoption by an accretion funnel, which would cause a hardening of the X-ray absorption. This is not observed, but the limit on extra absorption is weak due to the low count rates.

If taken seriously, the relation between X-ray luminosity and $\mathrm{H} \alpha$ absorption is consistent with CTTS being on average less luminous in X-rays than WTTS (e.g. Preibisch et al. 2005; Telleschi et al. 2007b). Drake et al. (2009) suggested that it is not the accretion of the CTTS that causes their low luminosity but rather, the low luminosity that allows the disk to approach the star more closely, i.e., weaker emitters to accrete and become CTTS.

\subsection{IM Lup in context}

Only a small number of CTTS have been observed with X-ray grating spectroscopy, most of them exhibiting low $f / i$ ratios in the He-like triplets, which T Tau does not. Does IM Lup represent a CTTS population with low densities such as T Tau or does it behave more like a WTTS? Although the complex $\mathrm{H} \alpha$ line profile is indicative of mass accretion, IM Lup differs from $\mathrm{T}$ Tau in other characteristics. The main component of the multiple system T Tau is much more massive, close to the boundary to the HerbigAe/Be stars, and $\mathrm{T}$ Tau accretes at much higher rates. Whereas IM Lup's X-ray emission must be coronal, T Tau could produce a significant part of its luminosity by accretion. We therefore expect IM Lup to resemble both WTTS and young MS stars in its X-ray properties. Only a few WTTS have been observed with X-ray gratings, but they all look similar to MS stars. Despite the small number observed, it appears credible that their emission is driven exclusively by a solar-type corona and we have found no evidence that the transition object IM Lup, which is surrounded by a disk, is different. The transition from a CTTS to a WTTS in X-rays seems to coincide with the decline of accretion as expected from accretion shock models for CTTS. However, the Ne IX triplet is the strongest discriminator and the original driver of the reported observation, but at the $90 \%$ confidence level the CTTS-like high-density scenario cannot be excluded for IM Lup.

\section{Summary}

We have presented a deep Chandra/HETGS grating spectrum of IM Lup, a WTTS with a circumstellar disk, and accompanying optical spectroscopy, some of which was obtained simultaneously to the X-ray spectra. IM Lup is an active star with an IFIP effect in a hot corona. The Ne IX He-like triplet represents the low-density limit, but due to the low signal we also cannot exclude a low $f / i$ scenario. It seems that the X-ray signatures of CTTS, i.e., a soft excess and high-density He-like triplets, are not correlated with the presence of the disk, but instead the active accretion of CTTS. The optical spectra of IM Lup exhibit emission lines that are typical of chromospherically active stars. This is consistent with the high X-ray luminosity of IM Lup.
However, the $\mathrm{H} \alpha$ line profiles are more complex than expected for MS chromospheres. This may be due to weak accretion funnels, such as those in CTTS with a low accretion rate.

Acknowledgements. CHIANTI is a collaborative project involving the NRL (USA), RAL (UK), MSSL (UK), the Universities of Florence (Italy) and Cambridge (UK), and George Mason University (USA). We made use of observations made with ESO Telescopes at the La Silla Observatory under programme ID 081.C-0779(A). The authors would like to thank Ian Waite, USQ, for the initial extraction of the IM Lup data from ANU. H.M.G. acknowledges support from DLR under 50OR0105. S.P.M. was supported by an appointment to the NASA Postdoctoral Program at Ames Research Center, administered by Oak Ridge Associated Universities through a contract with NASA. Z.-Y.L. acknowledges support from Chandra grant GO9-0007X.

\section{References}

Argiroffi, C., Maggio, A., Peres, G., Stelzer, B., \& Neuhäuser, R. 2005, A\&A, 439, 1149

Argiroffi, C., Maggio, A., \& Peres, G. 2007, A\&A, 465, L5

Arnaud, K. A. 1996, in Astronomical Data Analysis Software and Systems V, ASP Conf. Ser., 101, 17

Bagnulo, S., Jehin, E., Ledoux, C., et al. 2003, The Messenger, 114, 10 Baldovin-Saavedra, C., Audard, M., Duchêne, G., et al. 2009, ApJ, 697, 493 Balucinska-Church, M., \& McCammon, D. 1992, ApJ, 400, 699

Baraffe, I., Chabrier, G., Allard, F., \& Hauschildt, P. H. 1998, A\&A, 337, 403

Cabrit, S., Edwards, S., Strom, S. E., \& Strom, K. M. 1990, ApJ, 354, 687

Coffey, D., Bacciotti, F., \& Podio, L. 2008, ApJ, 689, 1112

D’Antona, F., \& Mazzitelli, I. 1994, ApJS, 90, 467

Dere, K. P., Landi, E., Mason, H. E., Monsignori Fossi, B. C., \& Young, P. R. 1997, A\&AS, 125, 149

Donati, J., Semel, M., Carter, B. D., Rees, D. E., \& Collier Cameron, A. 1997, MNRAS, 291, 658

Drake, J. J., Ercolano, B., Flaccomio, E., \& Micela, G. 2009, ApJ, 699, L35

Fang, M., van Boekel, R., Wang, W., et al. 2009, A\&A, 504, 461

Feigelson, E. D., \& Montmerle, T. 1999, ARA\&A, 37, 363

Flaccomio, E., Micela, G., \& Sciortino, S. 2003, A\&A, 402, 277

Fruscione, A., McDowell, J. C., Allen, G. E., et al. 2006, in SPIE Conf. Ser., 6270

Gabriel, A. H., \& Jordan, C. 1969, MNRAS, 145, 241

Gabriel, C., Denby, M., Fyfe, D. J., et al. 2004, in Astronomical Data Analysis Software and Systems (ADASS) XIII, ed. F. Ochsenbein, M. G. Allen, \& D. Egret, ASP Conf. Ser., 314, 759

Ghez, A. M., McCarthy, D. W., Patience, J. L., \& Beck, T. L. 1997, ApJ, 481, 378

Grevesse, N., \& Sauval, A. J. 1998, Space Sci. Rev., 85, 161

Güdel, M. 2004, A\&ARv, 12, 71

Güdel, M., \& Telleschi, A. 2007, A\&A, 474, L25

Güdel, M., \& Nazé, Y. 2009, A\&A Rev., 17, 309

Güdel, M., Briggs, K. R., Arzner, K., et al. 2007a, A\&A, 468, 353

Güdel, M., Skinner, S. L., Mel'Nikov, S. Y., et al. 2007b, A\&A, 468, 529

Günther, H. M., \& Schmitt, J. H. M. M. 2009, A\&A, 494, 1041

Günther, H. M., Liefke, C., Schmitt, J. H. M. M., Robrade, J., \& Ness, J.-U. 2006, A\&A, 459, L29

Günther, H. M., Schmitt, J. H. M. M., Robrade, J., \& Liefke, C. 2007, A\&A, 466, 1111

Hauschildt, P. H., \& Baron, E. 1999, Journal of Computational and Applied Mathematics, 109, 41

Huenemoerder, D. P., Kastner, J. H., Testa, P., Schulz, N. S., \& Weintraub, D. A. 2007, ApJ, 671, 592

Hughes, J., Hartigan, P., \& Clampitt, L. 1993, AJ, 105, 571

Hughes, J., Hartigan, P., Krautter, J., \& Kelemen, J. 1994, AJ, 108, 1071

Jayawardhana, R., Mohanty, S., \& Basri, G. 2003, ApJ, 592, 282

Kastner, J. H., Huenemoerder, D. P., Schulz, N. S., Canizares, C. R., \& Weintraub, D. A. 2002, ApJ, 567, 434

Kastner, J. H., Huenemoerder, D. P., Schulz, N. S., et al. 2004, ApJ, 605, L49

Lamzin, S. A. 1998, Astron. Rep., 42, 322

Landi, E., Del Zanna, G., Young, P. R., et al. 2006, ApJS, 162, 261

Liefke, C., Ness, J., Schmitt, J. H. M. M., \& Maggio, A. 2008, A\&A, 491, 859

Muzerolle, J., Calvet, N., \& Hartmann, L. 1998, ApJ, 492, 743

Muzerolle, J., Calvet, N., \& Hartmann, L. 2001, ApJ, 550, 944

Muzerolle, J., Hillenbrand, L., Calvet, N., Briceño, C., \& Hartmann, L. 2003, ApJ, 592, 266

Natta, A., Testi, L., Muzerolle, J., et al. 2004, A\&A, 424, 603

Ness, J.-U., Güdel, M., Schmitt, J. H. M. M., Audard, M., \& Telleschi, A. 2004, A\&A, 427, 667 
A\&A 519, A97 (2010)

Ness, J.-U., \& Wichmann, R. 2002, Astron. Nachr., 323, 129

Padgett, D. L., Cieza, L., Stapelfeldt, K. R., et al. 2006, ApJ, 645, 1283

Panić, O., Hogerheijde, M. R., Wilner, D., \& Qi, C. 2009, A\&A, 501, 269

Pinte, C., Padgett, D. L., Ménard, F., et al. 2008, A\&A, 489, 633

Porquet, D., Mewe, R., Dubau, J., Raassen, A. J. J., \& Kaastra, J. S. 2001, A\&A, 376, 1113

Preibisch, T., Kim, Y.-C., Favata, F., et al. 2005, ApJS, 160, 401

Reipurth, B., Pedrosa, A., \& Lago, M. T. V. T. 1996, A\&AS, 120, 229

Robinson, R. D., Cram, L. E., \& Giampapa, M. S. 1990, ApJS, 74, 891

Robrade, J., \& Schmitt, J. H. M. M. 2007, A\&A, 473, 229

Schegerer, A., Wolf, S., Voshchinnikov, N. V., Przygodda, F., \& Kessler-Silacci, J. E. 2006, A\&A, 456, 535

Schisano, E., Covino, E., Alcalá, J. M., et al. 2009, A\&A, 501, 1013

Schmitt, J. H. M. M., Robrade, J., Ness, J.-U., Favata, F., \& Stelzer, B. 2005 , A\&A, 432, L35
Siess, L., Dufour, E., \& Forestini, M. 2000, A\&A, 358, 593

Stelzer, B., \& Schmitt, J. H. M. M. 2004, A\&A, 418, 687

Telleschi, A., Güdel, M., Briggs, K. R., Audard, M., \& Palla, F. 2007a, A\&A, 468,425

Telleschi, A., Güdel, M., Briggs, K. R., Audard, M., \& Scelsi, L. 2007b, A\&A, 468,443

Telleschi, A., Güdel, M., Briggs, K. R., et al. 2007c, A\&A, 468, 541

Vuong, M. H., Montmerle, T., Grosso, N., et al. 2003, A\&A, 408, 581

White, R. J., \& Basri, G. 2003, ApJ, 582, 1109

Wichmann, R., Bastian, U., Krautter, J., Jankovics, I., \& Rucinski, S. M. 1998, MNRAS, 301, L39

Wichmann, R., Covino, E., Alcalá, J. M., et al. 1999, MNRAS, 307, 909

Worden, S. P., Schneeberger, T. J., \& Giampapa, M. S. 1981, ApJS, 46, 159 